\title{
Loss mechanisms in organic solar cells based on perylene diimide acceptors studied by time-resolved photoluminescence
}

\author{
Marina Gerhard*a, Dominik Gehrig ${ }^{\mathrm{b}}$, Ian A. Howard ${ }^{\mathrm{c}}$, Andreas P. Arndt ${ }^{\mathrm{d}}$, Mühenad Bilal ${ }^{\mathrm{a}}$, \\ Arash Rahimi-Iman ${ }^{\mathrm{a}}$, Uli Lemmer ${ }^{\mathrm{c}, \mathrm{d}}$, Frédéric Laquai ${ }^{\mathrm{b}, \mathrm{e}}$ and Martin Koch $^{\mathrm{a}}$ \\ ${ }^{a}$ Faculty of Physics and Material Sciences Center, Philipps-Universität Marburg, Renthof 5, D-35032 \\ Marburg, Germany; ${ }^{\mathrm{b}}$ Max Planck Research Group for Organic Optoelectronics, Max Planck Institute for \\ Polymer Research, Ackermannweg 10, 55128 Mainz, Germany; ' Institute of Microstructure Technology, \\ Karlsruhe Institute of Technology, Hermann-von-Helmholtz-Platz 1, D-76344 Eggenstein-Leopoldshafen, \\ Germany; ${ }^{\mathrm{d}}$ Light Technology Institute, Karlsruhe Institute of Technology, Kaiserstrasse 12, D-76131 \\ Karlsruhe, Germany; ${ }^{~}$ King Abdullah University of Science and Technology (KAUST), Solar and \\ Photovoltaics Engineering Research Center (SPERC), Thuwal 23955-6900, Kingdom of Saudi Arabia
}

\begin{abstract}
In organic photovoltaics (OPV), perylene diimide (PDI) acceptor materials are promising candidates to replace the commonly used, but more expensive fullerene derivatives. The use of alternative acceptor materials however implies new design guidelines for OPV devices. It is therefore important to understand the underlying photophysical processes, which either lead to charge generation or geminate recombination. In this contribution, we investigate radiative losses in a series of OPV materials based on two polymers, P3HT and PTB7, respectively, which were blended with different PDI derivatives. Our time-resolved photoluminescence measurements (TRPL) allow us to identify different loss mechanisms by the decay characteristics of several excitonic species. In particular, we find evidence for unfavorable morphologies in terms of large-scale pure domains, inhibited exciton transport and incomplete charge transfer. Furthermore, in one of the P3HT-blends, an interfacial emissive charge transfer (CT) state with strong trapping character is identified.
\end{abstract}

Keywords: Organic solar cells, perylene-diimide acceptors, exciton diffusion, charge transfer states

\section{INTRODUCTION}

Among electron accepting materials for organic photovoltaics, small molecule acceptors such as perylene diimides (PDIs) have attracted considerable interest, as they represent a low-cost alternative to the commonly used fullerene derivatives, such as PCBM. Fullerenes are difficult to functionalize for solution-processed device fabrication and therefore impede broader applications, whereas PDIs have been used as industrial dyes for many years. PDI absorption overlaps well with the solar spectrum, hence PDIs contribute efficiently to the light harvesting in OPV materials. Furthermore, the electron accepting and transport properties are beneficial for OPV applications and it is easy to tune their physicochemical properties, such as solubility or molecular packing, by introducing different side groups. ${ }^{1,2}$

However, replacing the fullerenes in organic solar cells by alternative acceptors is not trivial and it is unclear at present, why the efficiencies of non-fullerene solar cells generally lack behind those of fullerene-based devices, for which photoconversion efficiencies beyond $10 \%$ have been reported. ${ }^{3-5}$ Some of the major drawbacks of PDIs are probably their tendency to form large-scale pure aggregates in blends with donor molecules. Aggregation can be beneficial, when

Organic Photonics VII, edited by David Cheyns, Pierre M. Beaujuge, Volker van Elsbergen, Jean-Charles Ribierre, Proc. of SPIE Vol. 9895, 98950H · (c) 2016 SPIE

CCC code: $0277-786 X / 16 / \$ 18 \cdot$ doi: $10.1117 / 12.2227672$ 
the domain sizes do not exceed the length scale of exciton diffusion. In fact, for high-performance polymer:fullerene blends, the experimentally determined domain sizes correspond well with the exciton diffusion length on the order of $10 \mathrm{~nm} .{ }^{6}$ In case of planar PDI, however, morphologies with micrometer-sized crystallites have been reported for several blend material systems. ${ }^{7-10}$ Furthermore, there is experimental evidence that charge generation in PDI-based solar cells is limited by geminate losses at the interfaces. Interfacial CT states are assumed to play a key role in the charge generation process, as they may either dissociate into free charges or give rise to geminate recombination, thereby reducing the photoconversion efficiency (PCE). Temperature-dependent ultrafast vibrational spectroscopy on a P3HT:PDI blend has evidenced that exciton dissociation from the interface is a thermally activated process, whereas it has been found to be barrier-free in case of P3HT:PCBM. ${ }^{11}$ Geminate losses in a low-bandgap polymer:PDI-blend have also been identified by means of transient absorption spectroscopy. This finding agrees well with the observed field-dependent photocurrent yield, which has been investigated in the same study. ${ }^{8}$ A promising strategy to overcome the above-mentioned morphological drawbacks is the synthesis of PDI-derivatives employing structures, which effectively impede large-scale aggregation. For example, reduced aggregation has been demonstrated for a PDI derivative with core-alkylated side chains. ${ }^{12}$ Furthermore, smaller domain sizes have been successfully realized with twisted dimers. ${ }^{13-18}$ In this context also the rational selection of donor polymers and fine-tuning their properties have proven successful. ${ }^{15,16}$ The steady progress in the field of PDI-based OPV materials recently yielded a PCE exceeding $7 \%$ for a twisted dimer. ${ }^{18}$ This motivates further research, not only in terms of exploring and optimizing novel material systems, but also a detailed understanding of the underlying mechanisms of charge generation and the accompanying loss channels is desirable.

TRPL spectroscopy is an instructive tool to complement morphological studies on OPV material systems. It can be used to probe the subset of radiative excitons, which is not quenched at internal interfaces, thereby revealing information about the exciton diffusion process in pure domains of the donor or acceptor component. ${ }^{6,19}$ It has also been employed to detect radiative, interfacial CT states. ${ }^{20-22}$ In the work presented here, we investigate loss mechanisms in PDI-blends based on two polymers, P3HT and PTB7, respectively, by means of TRPL. Beside commercially available PDI, hereinafter referred as PDI-1, the polymers were blended with a core-alkylated PDI-derivative (PDI-2) and a PDI-dimer (PDI-3), providing six different blend films for investigation. In addition, we study pristine films of each material in order to obtain a more sophisticated picture of the intermixing effects.

This contribution is structured as follows: First, we will analyze spectral data in order to identify the excitonic species, which dominate the PL emission in the respective blend films. The impact of intermixing will be further investigated in a more sophisticated analysis of the PL dynamics. After this general overview, we will discuss in greater detail two interesting observations: (i) In PTB7-blends, we find evidence for inhibited exciton transport and incomplete charge transfer from the polymer domains, and (ii) P3HT:PDI-2 blends exhibit a spectral signature which we attribute to an interfacial CT state. We also reported on CT emission in our previous study on P3HT blended with the fullerene derivative $\mathrm{PC}_{61} \mathrm{BM}^{21}$ However, the thermal quenching characteristics of the CT state in P3HT:PDI-2 differs strongly from the findings for fullerene-based materials, therefore the last part will include a comparison and discussion of the temperature-dependent CT characteristics in the PDI and the fullerene-based blend.

\section{EXPERIMENTAL METHODS}

\subsection{Sample fabrication}

P3HT (Sepiolid P200) was purchased from BASF and PTB7 from 1-Material, respectively. The different PDI acceptor materials (Figure 1) were provided by the group of Prof. Müllen at the Max Planck Institute for Polymer Research, Mainz. Both, pristine films of each material and donor-acceptor blends were spin cast on glass substrates from chloroform solution with $1000 \mathrm{rpm}$. The concentration of the neat polymer solution was $10 \mathrm{mg} / \mathrm{ml}$. The PDIs were 
embedded in a passive polystyrene (PS) matrix for optical investigation with a concentration of $10 \mathrm{mg} / \mathrm{ml}$ of the respective solution. The materials for all blend films were dissolved with a concentration of $15 \mathrm{mg} / \mathrm{l}$ with a polymer:PDI ratio of $1: 2$.

Optical attenuation spectra were recorded using an optical spectrum analyzer and a tungsten white light source. Film thicknesses were determined with a profilometer, as summarized in Table 1. For electronic characterization of the PTB7blends, devices were fabricated from a second batch of films, similar to those described above, and J/V-curves were recorded in a solar simulator. The resulting PCEs are listed in Table 1.

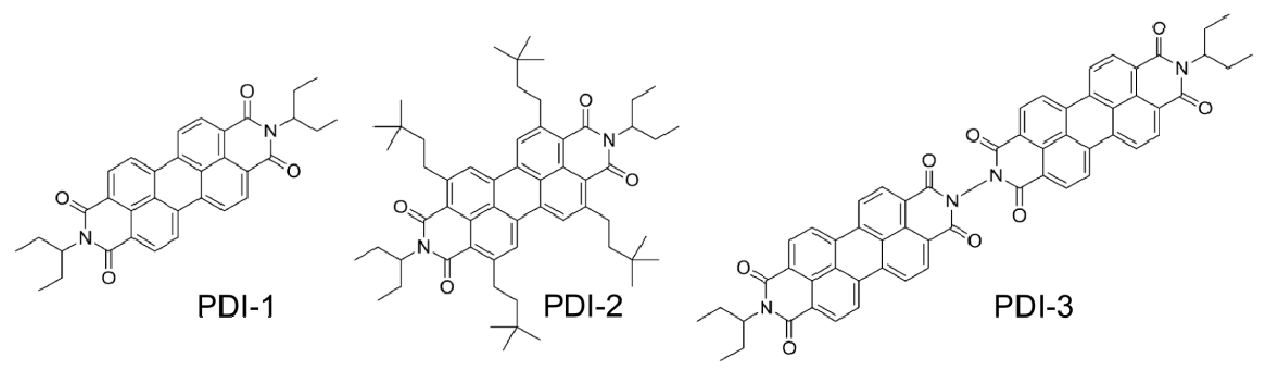

Figure 1. Chemical structures of the employed PDI-derivatives and nomenclature used in this contribution. The PDI-1 structure corresponds to commercially available PDI, the structure of PDI-2 comprises alkyl groups at the bay positions and PDI-3 represents the dimer.

Table 1. Film thicknesses and photoconversion efficiencies of the investigated polymer, PDI and blend films. The PCE values of the P3HT-blends are taken from Ref. 12, in which a donor:acceptor ratio of 1:1 was used for the blend films.

\begin{tabular}{ccc}
\hline Layer & Thickness / nm & PCE / \% \\
\hline PTB7 & 149 & -- \\
P3HT & 370 & -- \\
\hline PS:PDI-1 & 240 & -- \\
PS:PDI-2 & 241 & -- \\
PS:PDI-3 & 210 & -- \\
\hline PTB7:PDI-1 & 154 & 0.60 \\
PTB7:PDI-2 & 141 & 0.73 \\
PTB7:PDI-3 & 165 & 1.55 \\
\hline P3HT:PDI-1 & 125 & $0.25^{12}$ \\
P3HT:PDI-2 & 144 & $0.29^{12}$ \\
P3HT:PDI-3 & 142 & \\
\hline
\end{tabular}

\subsection{Time-resolved photoluminescence}

For TRPL studies, we used a Hamamatsu streak camera C5680 equipped with a synchroscan unit and an S1 cathode with a sensitivity range extending to the near infrared. The time resolution of the experiment mainly depends on the instrument's aperture sizes and the operated time window. For the measurements presented here, we applied time windows of $800 \mathrm{ps}$ and $2000 \mathrm{ps}$, yielding time resolutions of approximately $10 \mathrm{ps}$ and $35 \mathrm{ps,} \mathrm{respectively.} \mathrm{For} \mathrm{excitation}$ of the samples, we used a tunable titanium:sapphire oscillator (Spectra Physics) with an output wavelength of $920 \mathrm{~nm}$, pulse duration of $100 \mathrm{fs}$ and repetition rate of $80 \mathrm{MHz}$. The laser output was frequency doubled with a lithium triborate crystal, resulting in an excitation wavelength of $460 \mathrm{~nm}$. 


\section{RESULTS AND DISCUSSION}

Optical attenuation spectra of the blends and pristine films in the visible range and their PL spectra are summarized in Figure 2 and Figure 3, respectively. In order to elucidate the intermixing effects, the blend PL spectra in Figure 3 are plotted together with the time-integrated and normalized PL spectra of their respective donor and acceptor material. For all measurements, an excitation wavelength of $460 \mathrm{~nm}$ was chosen, allowing for excitation of both the donor and acceptor component.
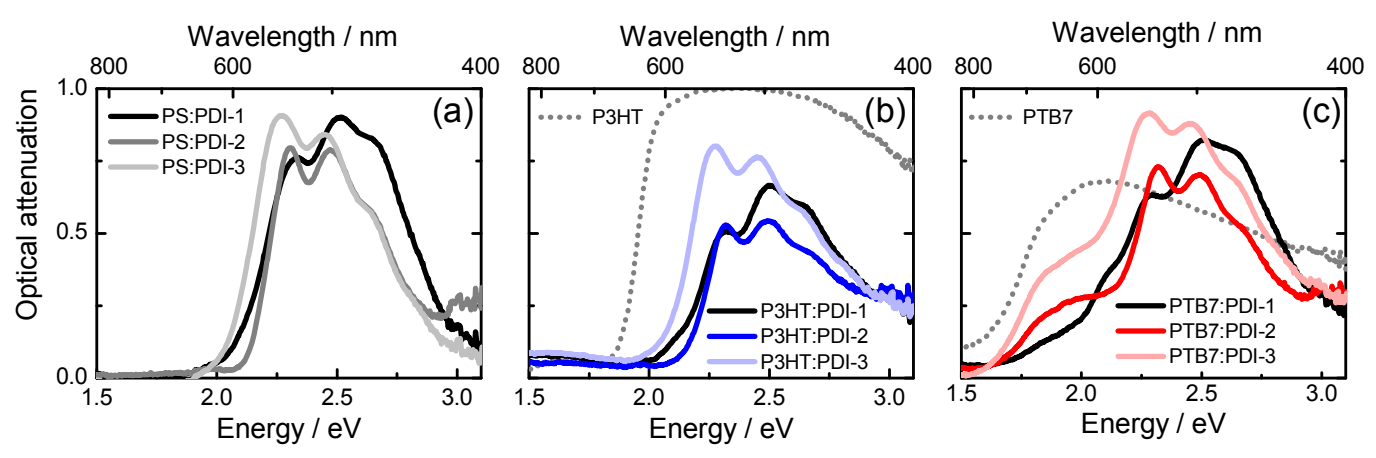

Figure 2. Optical attenuation spectra of (a) the three investigated PDIs embedded in polystyrene (PS), (b) blends of the PDIs with P3HT and a pristine P3HT film for comparison and (c) blends of the PDIs with PTB7 and a pristine PTB7-film, respectively.

In case of the P3HT blends (upper row of panels in Figure 3), all investigated films exhibit PL signatures in the spectral range of the PDI emission but no contribution similar to the PL spectrum of pristine P3HT is observed. This could be due to the fact that P3HT is intimately mixed with the PDI molecules, implying that P3HT excitons would not have to diffuse to an interface before they are quenched. Such ultrafast quenching channels on a sub-ps timescale have been demonstrated in TRPL studies on polymer:fullerene blends, for example, on P3HT:PC ${ }_{61} \mathrm{BM}^{19,21}$ and $\mathrm{PTB} 7: \mathrm{PC}_{71} \mathrm{BM}^{6,22}$ and there is evidence that the occurrence of this mechanism is correlated with the degree of intermixing on a molecular scale. Another reason for the absence of characteristic P3HT signatures could be substantial changes of the P3HT conformation in blends with PDI. In previous work, it has been pointed out that P3HT crystallization is strongly inhibited in P3HT:PDI-2 ${ }^{12}$, as indicated by the absence of certain absorption bands around $2 \mathrm{eV}$ in the blend spectra, which are assigned to P3HT aggregates. The absorption curves we measured for the P3HT blends corroborate these findings, also in case of P3HT:PDI-1 and P3HT:PDI-3. The presumable change in conformation could also lead to a blue-shift of the PL emission, ${ }^{23}$ hence the PL signature of the P3HT:PDI blends could have contributions from both PDI and P3HT emission. Another interesting observation is the long-lived emission peaking around $1.4 \mathrm{eV}$, which exclusively occurs in P3HT:PDI-2. The signature deviates clearly from the polymer and PDI emission and is significantly red-shifted with respect to them, therefore it appears reasonable to attribute it to an interfacial CT state. We will return to a more detailed discussion of its dynamics later.

A different situation arises for the PTB7:PDI blends (lower row in Figure 3), where we identify signatures of both PTB7 and PDI excitons in the PL spectra. For all investigated PTB7-samples, the PL emission of PTB7 is stronger than the PDI emission, although the films were fabricated with excess PDI and the absorption curves (see Figure 2.c) indicate that for $460 \mathrm{~nm}$ a higher amount of photons is absorbed in the PDI domains. The discrepancy in the PL intensity ratios could be due to Förster resonance energy transfer from PDI to PTB7. As the emission spectra of the PDIs overlap to a large extent with the PTB7 absorption range, this process is likely to occur. In previous work on PBDTTT-C:PDI-1, where the 
polymer absorption is similar to that of PTB7, the Förster transfer rate has been estimated to the inverse of 3 ps, ${ }^{8}$ which is below the time resolution of our experiment. Another reason for the higher PL yield of the PTB7 component could be inhibited quenching of PTB7 excitons at donor-acceptor interfaces, which we assume, is in particular the case for the PTB7:PDI-2 blend. We will further elaborate this hypothesis in connection with a more detailed discussion on the PL dynamics in the next paragraph.
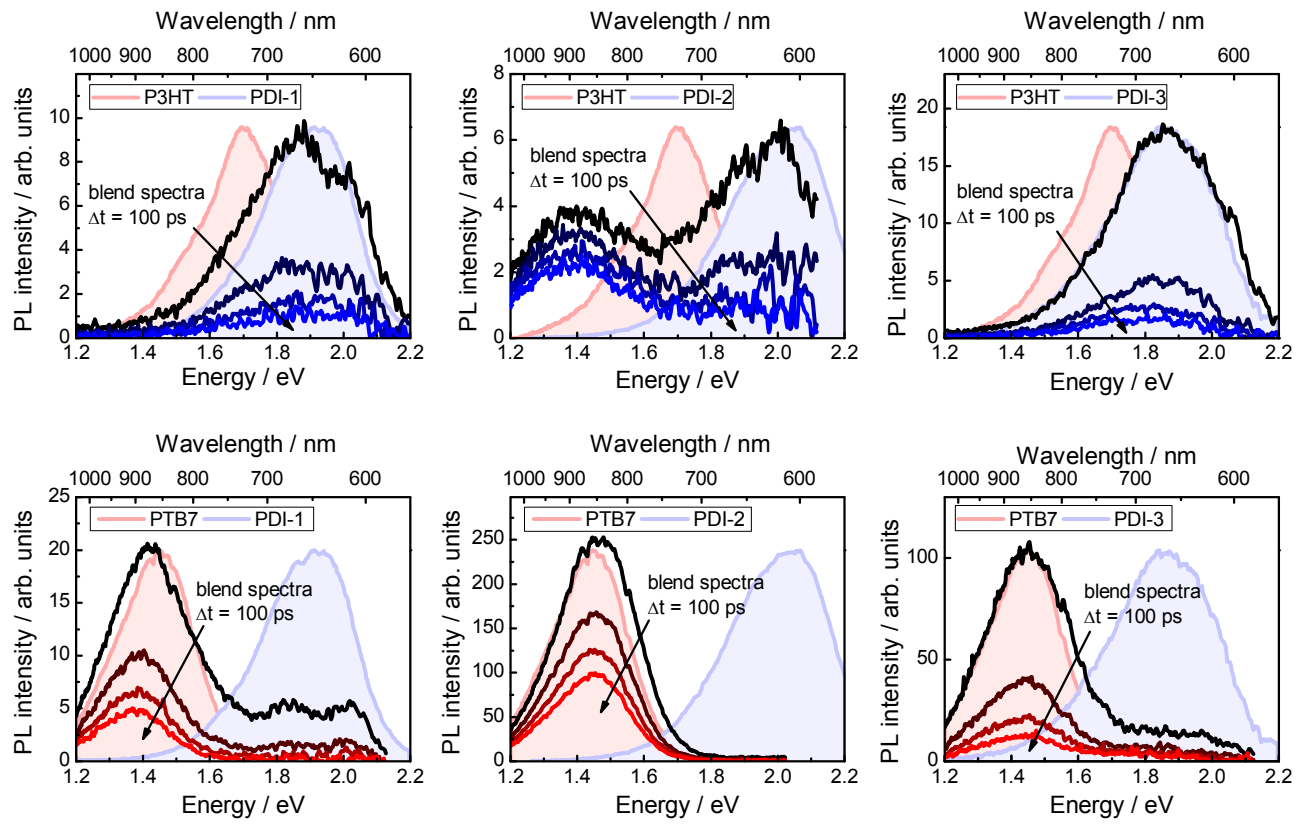

Figure 3. Comparison of the PL spectra of pristine and blend films for the six investigated heterojunctions. Each plot shows the time-integrated and normalized spectra of pristine donor and acceptor material (filled curves in the background) and the spectral decay characteristics of their respective blend, as labelled. The blend spectra are shown for different linearly progressing time intervals after optical excitation between $0-100 \mathrm{ps}$, with the direction of time-evolution indicated by arrows.

Further insight into the intermixing effects is provided by the PL dynamics. The PL in the blends is expected to decay faster, when interfacial quenching competes effectively with radiative recombination. As already indicated by the timedependent PL spectra in Figure 3, the PL decay characteristics of the blends are complex, according to the contributions of several excitonic species. Therefore, we will treat the high $(1.8-2.1 \mathrm{eV})$ and the low $(1.3-1.6 \mathrm{eV})$ energy ranges of the PL emission separately, corresponding to the energy intervals which are dominated by the PL emission of the PDI and the polymer, respectively.

In the energy range between 1.8 and $2.1 \mathrm{eV}$, we probe the PDI emission, as presented in Figure 4.a and Figure 4.c. The PDI signatures in the blends exhibit faster decay than those in the pristine films. For the PDI signatures in the blends, we measure 1/e-decay times of 20-50 ps, which is similar to previous results on PBDTTT-C:PDI-1, where time constants of 20-30 ps have been assigned to diffusion-related exciton quenching in PDI domains by means of transient absorption spectroscopy. ${ }^{8}$ Compared to the pristine PDI films, the decay constants are decreased by factors ranging between 3 in case of the PDI-1 blends and 14 for P3HT:PDI-2 and P3HT:PDI-3, indicating that significant portions of the PDI population can be quenched either via hole or exciton transfer after diffusion to an interface. The lower quenching efficiency of the PDI-1 blends can be explained in terms of an enhanced tendency of the PDI-1 molecules to aggregate, triggering the formation of more extended domains with higher diffusive losses. This is supported by the absorption 
curves of PDI-1 in PS and the PDI-1 blends (Figure 2), as they show a significant decrease of the 0-0 transition at $2.3 \mathrm{eV}$ with respect to the $0-1$ transition at $2.5 \mathrm{eV}$, which is typically observed in case of $\mathrm{H}$-aggregates ${ }^{12,24}$. Furthermore, these stabilized intermolecular states are likely to act as traps for diffusive excitons. ${ }^{7}$

In case of P3HT:PDI-1 and P3HT:PDI-3, the low energy range of the PL emission between 1.3 and $1.6 \mathrm{eV}$ (Figure 4.b) shows essentially the same decay characteristics as the high-energy part, indicating, that this range of the spectra is governed by PDI emission as well. In case of P3HT:PDI-2, however, the PL decays even slower than in the pristine P3HT film. This slow decay is attributed to the presumable CT emission around $1.4 \mathrm{eV}$ (see Figure 3). This assignment is supported by the monoexponential decay dynamics, because it appears unlikely that the emissive and localized CT excitons undergo diffusion or spectral relaxation, which would imply more complex decay characteristics. The PL decay of the PTB7-blends in the range between 1.3 and $1.6 \mathrm{eV}$ (Figure 4.d) is dominated by the polymer emission. From a comparison of the 1/e-decay times we find that the emission of PTB7:PDI-1 $\left(\tau_{1 / e}=50 \mathrm{ps}\right)$ and PTB7:PDI-3 $\left(\tau_{1 / e}=35 \mathrm{ps}\right)$ decays by a factor of 2-3 faster than the emission of the pristine film $\left(\tau_{1 / e}=100 \mathrm{ps}\right)$, indicating that a diffusion-related quenching mechanism is present, most likely interfacial electron transfer from the polymer to PDI. Again, the observed time-scale of exciton quenching is similar to previous work on PBDTTT-C:PDI- $1 .^{8}$
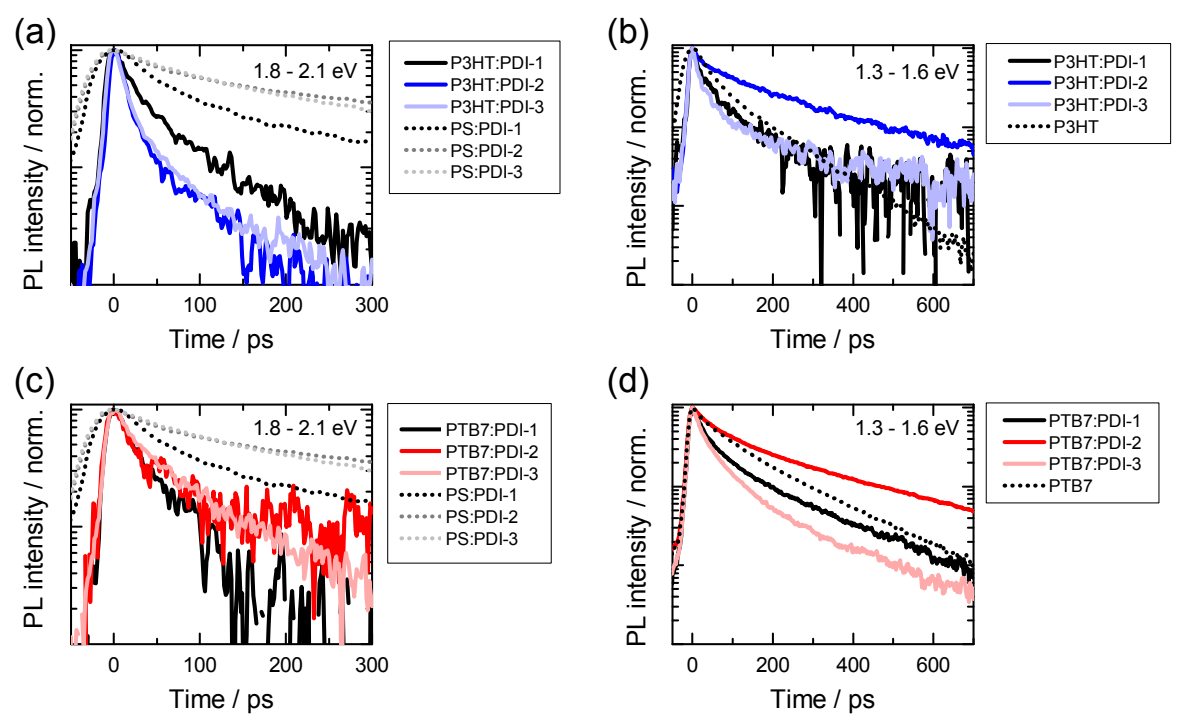

Figure 4. Normalized PL transients for the P3HT-films (upper row) and for the PTB7-films (lower row). Panels (a) and (c) represent transients integrated over the high-energy range of the PL spectra between 1.8 and $2.1 \mathrm{eV}$, corresponding to the PDI emission. Panels (b) and (d) show the PL decay in the energy range between 1.3 and $1.6 \mathrm{eV}$, which is dominated by the polymer and presumable CT emission, respectively. For the blend films and PTB7, the streak camera was operated using a time range of $800 \mathrm{ps}$, whereas the data for pristine PDI films and P3HT was recorded with a $2000 \mathrm{ps}$ time window.

For the PTB7:PDI-2 blend, however, the PTB7-signature decays even slower than in the pristine film. Therefore it appears unlikely, that charges are efficiently transferred from PTB7 to PDI-2. This result is surprising, because the energy level offset between the donor and acceptor LUMOs should actually provide an efficient driving force for CT reactions. However, unfavorable molecular packing could significantly limit this process. This could be either due to large distances between donor and acceptor molecules, limiting the Marcus transfer rate, or because intermixing with PDI molecules affects the exciton transport to the interfaces within the polymer matrix. More precisely, molecularly dispersed PDI could interpenetrate the polymer matrix, thereby increasing the average distance of the chromophores, 
which would in turn decrease the diffusion efficiency of PTB7 excitons. In fact, we find evidence for inhibited spectral diffusion in PTB7:PDI-2, when we compare the spectral relaxation characteristics to that of pristine PTB7. The maximum of the PTB7 emission in the blend with PDI-2 is found to exhibit only a small red shift of $25 \mathrm{meV}$, whereas the emission of the pristine film red-shifts by approximately $50 \mathrm{meV}$ within the observed time window of $2 \mathrm{~ns}$ (not shown here). This indicates higher exciton transfer rates between the chromophores in the pristine polymer film, as the excitons can more efficiently energetically relax into local minima within the density of states.

In the PTB7:PDI-1 and the PTB7:PDI-3 blends on the other hand, the red-shifting of the PL emission in time is stronger than for pristine PTB7, which is accompanied by spectral narrowing and an increase of the PL lifetimes at lower emission energies. It appears, however, unlikely that this is due to enhanced exciton diffusion in the PTB7 matrix, as intermixing with small molecules should rather hamper this process. When interfacial quenching is present, two other mechanisms could account for the observed spectral red shift in time: Either preferential charge transfer from energetically higher excitonic states or the transfer of the polymer excitonic population into a CT state. Within the given framework of methods, this issue cannot be entirely resolved. However, no clear two-component decay, as expected for superimposed singlet and CT emission, is observed for the PTB7:PDI-1 and the PTB7:PDI-3 decay and their spectral signatures are not far red-shifted from the original PTB7 emission. Furthermore, their long-lived decay constants are similar to the decay of the pristine PTB7 film. Therefore, it appears more likely to assume that the observed PL signatures are in fact entirely present due to recombination in the polymer and only a subset of the PTB7 excitons undergoes interfacial quenching, whereas the quenching probability depends on the exciton energy. This means, that excitons which occupy energetically higher states are more likely to be quenched, whereas excitons at lower energies rather undergo geminate recombination. With these assumptions, the observed PL characteristics corroborate the finding of time-delayed charge generation after exciton diffusion in the polymer-component by means of transient absorption spectroscopy. ${ }^{8}$

Finally, we investigate the effect of temperature on the PL emission in greater detail. Therefore, we select the P3HT:PDI-2 blend, as it allows us to study both exciton diffusion in PDI domains and the temperature dependence of the presumable CT emission. The results will be compared to our previous work on P3HT:PCBM. ${ }^{21}$ The temperaturedependent dynamics of the singlet states in both material systems are summarized in Figure 5.a-c. In both cases, thermal energy enhances the amount of PL quenching, which is attributed to faster diffusion of excitons to an interface (no significant change in the initial intensity was observed for the P3HT:PCBM blend). The CT signatures (Figure 5.d-f) on the other hand decay on longer time scales according to their more localized nature. Here, an important difference arises for the fullerene- and the PDI-based material: In P3HT:PCBM, a significant amount (65\%) of the CT intensity is quenched at room temperature, compared to the intensity at $10 \mathrm{~K}$, whereas no noticeable change in intensity is observed for the signature in P3HT:PDI-2. Thermally activated CT quenching has also been observed in other polymer:fullerene systems. ${ }^{20,22}$ It is important to point out that this thermally-activated CT quenching occurs to a large extent on a short timescale, whereas the long-lived decay component of the CT emission is not essentially altered. Hence, in the fullerenebased materials, the long-lived emission should be attributed to a trapping process, but there is a thermally activated mechanism, which allows charges to bypass these trap states. This mechanism seems to be absent in the investigated PDI-blend. Actually, the CT intensity in P3HT:PDI-2 increases slightly with temperature, which could be due to a more efficient diffusion of PDI excitons to an interface. However, the rise time of the CT emission falls below the time resolution of our experiment, therefore it is unlikely that the CT states are preferentially populated from diffusive PDI excitons. In any case, the absence of "hot" bypassing channels for the emissive CT state within the charge generation process could seriously limit the efficiency of PDI-based devices. 
(a)

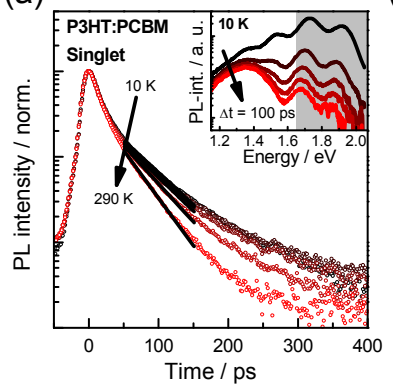

(d)

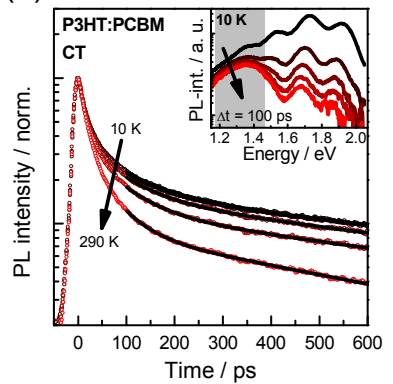

(b)

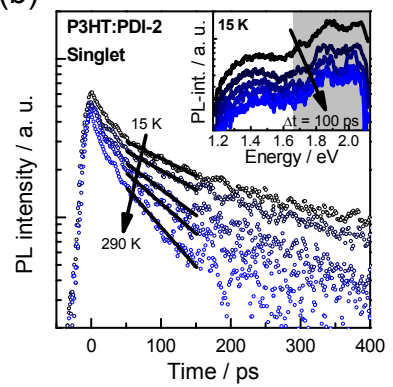

(e)

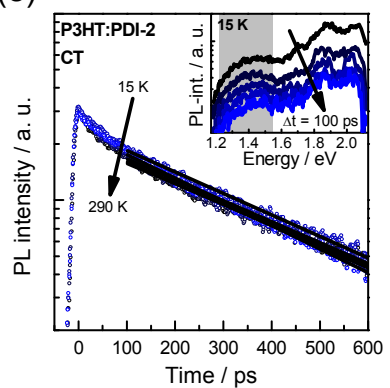

(c)

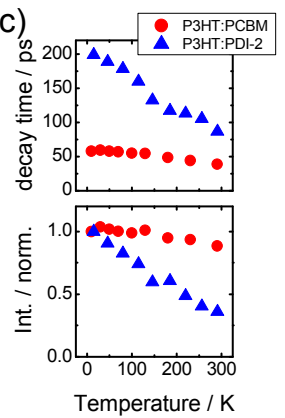

(f)

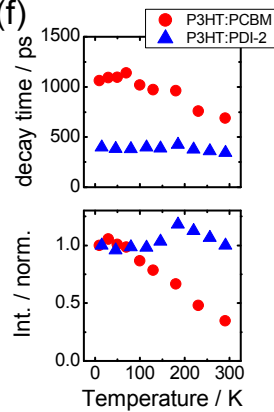

Figure 5. Results of temperature-dependent studies on the PL emission in P3HT:PCBM (panels (a) and (d); data from Ref. 21) and P3HT:PDI-2 (panels (b) and (e)). In case of P3HT:PCBM, the transients are normalized, because no clear correlation between temperature and PL intensity has been observed for the singlet states. The upper row summarizes the decay characteristics of the singlet states, which is obtained from the transients integrated over the highlighted regions of the spectra in the insets. In order to emphasize the complex decay characteristics, the spectra are presented for different time intervals after optical excitation. The depicted transients are exemplary data obtained for different temperatures between 10 and $290 \mathrm{~K}$. The lower row shows the temperature-dependent decay characteristics of the CT emission, which again is highlighted in the insets of panels (d) and (e). The decay times of the respective species are approximated with exponential fit functions, as highlighted by the thick black curves. The decay times of the emissive CT state in P3HT:PCBM were approximated with the longer-lived component of a biexponential model function, because the CT signature was superimposed by singlet emission. The extracted decay times are presented together with the corresponding PL intensities in panels (c) and (f), respectively.

\section{CONCLUSIONS}

In summary, we have characterized two series of non-fullerene OPV material systems, based on different PDI derivatives and the benchmark donor polymers P3HT and PTB7, by means of TRPL. Several origins of losses within the charge generation process have been identified. We have found strong evidence, that the PDI molecules alter the polymer matrix in several unfavorable ways. Both absorption and PL spectra indicate that P3HT crystallization is strongly suppressed in blends with PDI. However, there is a general consensus that P3HT crystallization is important to achieve reasonable performance in fullerene-based devices. The PDI molecules also seem to have an impact on the packing of the polymer in blends with PTB7, in particular for PTB7:PDI-2, we have found evidence for reduced diffusive exciton transport, resulting in a high PL yield and no noticeable CT. Incomplete charge transfer is also observed for the other PTB7:PDIblends, which corroborates previous findings of diffusion-limited charge generation in PBDTTT-C:PDI- $1 .{ }^{8}$ These findings outline the importance to control the nanomorphology and exciton hopping rates in these systems, in order to avoid geminate losses during the exciton diffusion process. Furthermore, the interfaces in polymer:PDI systems can play a critical role, as we point out by our temperature-dependent study of the CT emission in P3HT:PDI-2. In contrast to many fullerene-based material systems, we see no indication for a thermally activated bypassing mechanism, which could reduce the interfacial trapping of charges at room temperature. More efforts should be undertaken to analyze, under which circumstances interfacial trapping can be avoided, as this could significantly reduce the amount of losses. 


\section{ACKNOWLEDGEMENTS}

Financial support of this contribution from the German Academic Exchange Service (DAAD) is gratefully acknowledged.

\section{REFERENCES}

[1] Huang, C., Barlow S. and Marder, S. R., "Perylene-3,4,9,10-tetracarboxylic Acid Diimides: Synthesis, Physical Properties, and Use in Organic Electronics," J. Org. Chem. 76, 2386-2407 (2011).

[2] Shin, W. S., Jeong, H.-H., Kim, M.-K., Jin, S.-H., Kim, M.-R., Lee, J.-K., Lee, J. W. and Gal, Y.-S., "Effects of functional groups at perylene diimide derivatives on organic photovoltaic device application," J. Mater. Chem. 16, 384-390, (2006).

[3] Hu, H., Jiang, K., Yang, G., Liu, J., Li, Z., Lin, H., Liu, Y., Zhao, J., Zhang, J., Huang, F., Qu, Y., Ma, W. and Yan, H., "Terthiophene-Based D-A Polymer with an Asymmetric Arrangement of Alkyl Chains That Enables Efficient Polymer Solar Cells," J. Am. Chem. Soc. 137, 14149-14157 (2015).

[4] Green, M. A., Emery, K., Hishikawa, Y., Warta, W. and Dunlop, E. D., "Solar cell efficiency tables (version 47)," Prog. Photovolt: Res. Appl. 24, 3-11 (2015).

[5] Zhao, J., Li, Y., Yang, G., Jiang, K., Lin, H., Ade, H., Ma, W. and Yan, H., "Efficient organic solar cells processed from hydrocarbon solvents," Nature Energy 1, 15027 (2016).

[6] Hedley, G. J., Ward, A. J., Alekseev, A., Howells, C. T., Martins, E. R., Serrano, L. A., Cooke, G., Ruseckas, A. and Samuel, I. D. W. "Determining the optimum morphology in high-performance polymer-fullerene organic photovoltaic cells," Nat. comm. 4, 2867 (2013).

[7] Howard, I. A., Laquai, F., Keivanidis, P. E., Friend, R. H. and Greenham, N. C., "Perylene Tetracarboxydiimide as an Electron Acceptor in Organic Solar Cells: A Study of Charge Generation and Recombination," J. Phys. Chem. C 113, 21225-21232 (2009).

[8] Gehrig, D. W., Roland, S., Howard, I. A., Kamm, V., Mangold, H., Neher, D. and Laquai, F., "Efficiency-Limiting Processes in Low-Bandgap Polymer:Perylene Diimide Photovoltaic Blends," J. Phys. Chem. C 118, 20077-20085 (2014).

[9] Rajaram, S., Armstrong, P. B., Kim, B. J. and Fréchet, J. M. J., "Effect of Addition of a Diblock Copolymer on Blend Morphology and Performance of Poly(3-hexylthiophene):Perylene Diimide Solar Cells," Chem. Mater. 21, 1775-1777 (2009).

[10] Dittmer, J. J., Marseglia, E. A. and Friend, R. H., "Electron Trapping in Dye-Polymer Blend Photovoltaic Cells," Adv. Mater. 12, 1270-1274 (2000).

[11] Pensack, R. D., Guo, C., Vakhshouri, K., Gomez, E. D. and Asbury, J. B., "Influence of Acceptor Structure on Barriers to Charge Separation in Organic Photovoltaic Materials," J. Phys. Chem. C 116, 4824-4831 (2012).

[12] Kamm, V., Battagliarin, G., Howard, I. A., Pisula, W., Mavrinskiy, A., Li, C., Müllen, K. and Laquai, F., "Polythiophene:Perylene Diimide Solar Cells - the Impact of Alkyl-Substitution on the Photovoltaic Performance," Adv. Energy Mater. 1, 297-302 (2011). 
[13] Lu, Z., Jiang, B., Zhang, X., Tang, A., Chen, L., Zhan, C. and Yao, J., "Perylene-Diimide Based Non-Fullerene Solar Cells with 4.34 \% Efficiency through Engineering Surface Donor/Acceptor Compositions," Chem. Mater. 26, 2907-2914 (2014).

[14] Zhang, X., Jiannian, Y. and Zhan, C., "A selenophenyl bridged perylene diimide dimer as an efficient solutionprocessable small molecule acceptor," Chem. Commun. 51, 1058-1061 (2015).

[15] Ye, L., Jiang, W., Zhao, W., Zhang, S., Qian, D., Wang, Z. and Hou, J., "Selecting a Donor Polymer for Realizing Favorable Morphology in Efficient Non-fullerene Acceptor-based Solar Cells," Small 10, 4658-4663 (2014).

[16] Zhao, J., Li, Y., Lin, H., Liu, Y., Jiang, K., Mu, C., Ma, T., Lai, J. Y. L., Hu, H., Yu, D. and Yan, H., "Highefficiency non-fullerene organic solar cells enabled by a difluorobenzothiadiazole-based donor polymer combined with a properly matched small molecule acceptor," Energy Environ. Sci. 8, 520 (2015).

[17] Zhong, Y., Trinh, M. T., Chen, R., Wang, W., Khlyabich, P. P., Kumar, B., Xu, Q., Nam, C.-Y., Sfeir, M. Y., Black, C., Steigerwald, M. L., Loo, Y.-L., Xiao, S., Ng, F., Zhu, X.-Y. and Nuckolls, C., "Efficient Organic Solar Cells with Helical Perylene Diimide Electron Acceptors," J. Am. Chem. Soc. 136, 15215-15221 (2014).

[18] Sun, D., Meng, D., Cai, Y., Fan, B., Li, Y., Jiang, W., Huo, L., Sun, Y. and Wang, Z., "Non-Fullerene-AcceptorBased Bulk-Heterojunction Organic Solar Cells with Efficiency over 7 \%," J. Am. Chem. Soc. 137, 11156-11162 (2015).

[19] Piris, J., Dykstra, T. E., Bakulin, A. A., van Loosdrecht, P. H. M., Knulst, W., Trinh, M. T., Schins, J. M. and Siebbeles, L. D. A., "Photogeneration and Ultrafast Dynamics of Excitons and Charges in P3HT/PCBM Blends," $J$. Phys. Chem. C 113, 14500 (2009).

[20] Jarzab, D., Cordella, F., Gao, J., Scharber, M., Egelhaaf, H. -. J. and Loi, M. A., "Low-Temperature Behaviour of Charge Transfer Excitons in Narrow-Bandgap Polymer-Based Bulk Heterojunctions," Adv. Energy Mater. 1, 604 (2011).

[21] Arndt, A. P., Gerhard, M., Quintilla, A., Howard, I. A., Koch, M. and Lemmer, U., "Time-Resolved ChargeTransfer State Emission in Organic Solar Cells: Temperature and Blend Composition Dependence of Interfacial Traps," J. Phys. Chem. C 119, 13516-13523 (2015).

[22] Gerhard, M., Arndt, A. P., Howard, I. A., Rahimi-Iman, A., Lemmer, U. and Koch, M. ,"Temperature and EnergyDependent Separation of Charge-Transfer States in PTB7-Based Organic Solar Cells," J. Phys. Chem. C 119, 28309-28318 (2015).

[23] Hallermann, M., Kriegel, I., Da Como, E., Berger, J. M., von Hauff, E. and Feldmann, J.,"Charge Transfer Excitons in Polymer/Fullerene Blends: The Role of Morphology and Polymer Chain Conformation," Adv. Funct. Mater. 19, 3662-3668 (2009).

[24] Beckers, E. H. A., Meskers, S. C. J., Schenning, A. P. H. J., Chen, Z., Würthner, F., Marsal, P., Beljonne, D., Cornil, J. and Janssen, R. A. J., "Influence of Intermolecular Orientation on the Photoinduced Charge Transfer Kinetics in Self-Assembled Aggregates of Donor-Acceptor Arrays," J. Am. Chem. Soc. 128, 649-657 (2006). 